\title{
System for Supporting Web-based Public Debate Using Transcripts of Face-to-Face Meeting
}

\author{
Shun Shiramatsu ${ }^{1}$, Jun Takasaki ${ }^{1}$, Tatiana Zidrasco ${ }^{1}$, Tadachika Ozono ${ }^{1}$, \\ Toramatsu Shintani ${ }^{1}$, and Hiroshi G. Okuno ${ }^{2}$ \\ 1 Graduate School of Engineering, Nagoya Institute of Technology, \\ Gokisho-cho, Showa-ku, Nagoya, Japan \\ 2 Graduate School of Informatics, Kyoto University, \\ Yoshida-honmachi, Sakyo-ku, Kyoto, Japan \\ siramatu@nitech.ac.jp
}

\begin{abstract}
We propose a public debate support system through iterative alternation of face-to-face meetings and Web-based debates. Web-based facilitation of public debates is suitable for complementary question and answering when the debate in face-to-face meetings is insufficient due to time restriction. Since transcripts of public meetings tend to be lengthy and specialized, sharing public concerns among citizens and stakeholders requires much time and effort. To solve this problem, we propose two approaches. First, supporting the sharing of public concerns with structuration of public debate based on rhetorical structure theory (RST). It enables a user to manually specify his/her intention of a question, e.g., the question requires more evidence. An intention tag is effective for facilitating public debate. Second, supporting reading with visualization of topic transition, using our developed SalienceGraph. Moreover SalienceGraph retrieves passages related to a transient topic from past meeting records or documents. These approaches support citizens and stakeholders in finding, tracking, and sharing public concerns.
\end{abstract}

\section{Introduction}

We are developing a framework for supporting public debate to facilitate public involvement. Public involvement in building consensus for community development requires much effort and time for sharing public concerns among citizens and stakeholders. This is because understanding long transcripts of face-to-face public debates requires much time and effort for non-expert citizens. Moreover, Web-based asynchronous debate is suitable for complementary question and answering when the debate in face-to-face meetings is insufficient due to time restriction. Hence, our framework consists of iterative alternation of face-to-face meetings and Web-based debates.

Over the past few decades, there has been a growing debate about the role of the public in determining policy regarding health and environmental risk management [1. To build consensus through public involvement, aggregating and sharing opinions aired in public debates is important [23]. In this process,

N. García-Pedrajas et al. (Eds.): IEA/AIE 2010, Part III, LNAI 6098, pp. 311-320, 2010.

(C) Springer-Verlag Berlin Heidelberg 2010 
public concerns can be managed according to the spiral process for knowledge creation 4 by using meeting records as shown in Figure 1

For example, transcripts of the Yodo River Basin Committee, which include long public meetings, are published as lengthy PDF files on the Web [5]. Meetings in the committee have been held over 200 times and each meeting transcript has hundreds of sentences and several dozens pages. Although there are manual summaries, they are no effective way of finding an argument because the summaries cannot include all arguments of a debate.

Moreover, to reduce the amount of time and effort needed to understand the arguments, support for grasping the background of the arguments is needed. Since background information is often omitted by meeting participants, readers of meeting records often need to check relevant information. To complement ommited information, citizens need asynchronous question and answering and searching relevant information when they read the meeting transcripts. This study focuses on the rhetorical structure of a meeting to facilitate asynchronous question and answering.

This study deals with the following issues for supporting public debate.

(1) Facilitate asynchronous debate to complement insufficiency of face-to-face debate and to share public concerns included in meeting transcripts

(2) Provide an overview of transition of a long meeting to reduce the time and effort needed to track and find arguments

(3) Reduce the amount of time and effort needed to understand background or relevant information of arguments

To address issue (1), we have developed a Web-based interface for commenting an meeting transcripts based on rhetorical structure theory (RST). To address issue (2), we have developed a visualizer for transitions of a topic in a meeting based on a metric for focus of attention on each term in the meeting. Since joint attention among meeting participants and readers dynamically changes sentence by sentence, the metric is designed to deal with dynamic transition. To address issue (3), we have developed a method for retrieving information related to a transient topic based on the above-mentioned metric.

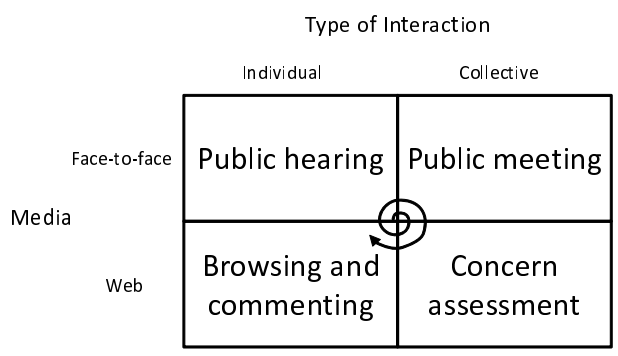

Fig. 1. Iterative alternation of face-to-face meetings and Web-based debates for sharing public concerns 


\section{Literature Reviews}

Malone et al. developed on-line debate system called Climate Collaboratorium [6] to harness the collective intelligence of citizens. Their aim is similar to ours, that is, complement debate to share public concerns (issue (1)). Although their main approach is to use user-created plans for debate, they do not focus on the rhetorical structure of a debate.

To enable users to track and find arguments from a long meeting (issue (2)), visualizing an overview of transition is more effective than conventional methods for visualizing arguments. There are several argument visualization tools [7, such as ArguMed [8], Araucaria [9], Semantic Editor [10], etc. Typically, these tools produce "box and arrow" diagrams in which premises and conclusions are formulated as statements [11. We have developed a method for visualizing the transitions of a topic because solving issue (2) requires an overview of transition of a long meeting.

Several debate browsers for a multimodal record of a meeting have been developed [12 13]. Furthermore, several environments for online argumentation based on "box and arrow" diagrams have also been developed [14 15]. Although we need to deal with multimodal meeting records and constructing argumentation environment is necessary, we focus on the transcription of a meeting record.

To help the user understand the background of arguments (issue (3)), our method for visualizing topic transition can be naturally expanded to a new method for finer-grain retrieval of information related to transient topics. This retrieval method does not require text segmentation. Several topic mixture methods for finer-grain information retrieval have been proposed [16 17]18. These conventional methods require text segmentation to deal with dynamic transition. Although these methods can be used to provide topic-related information for issue (3), we use a new method naturally expanded from our visualization method for issue (2).

Furthermore, solving issues (2) and (3) also supports a corpus-based analysis of public debate [19] because solving them can reduce the time and effort needed for researchers to read a long public debate.

\section{Web-based Interaction for Sharing Public Concerns}

To complement insufficiency in face-to-face meetings, we are developing a prototype called SalienceGraph, shown in Figure 2, Citizens can write comments about meeting transcripts into the right-side of the figure via the Web. Transcripts of face-to-face meetings are stored as Global Document Annotation (GDA) files to represent linguistic structure [20]. Comments written via Web are stored as Resource Description Framework (RDF) files to represent rhetorical structure.

The rhetorical relation tags between the comments and statements in the transcripts are manually assigned by users. Although the tag set is designed based on rhetorical structure theory (RST) 21], we introduce a set of novel tags representing intentions of questions, that is, require-evidence, require-example, 


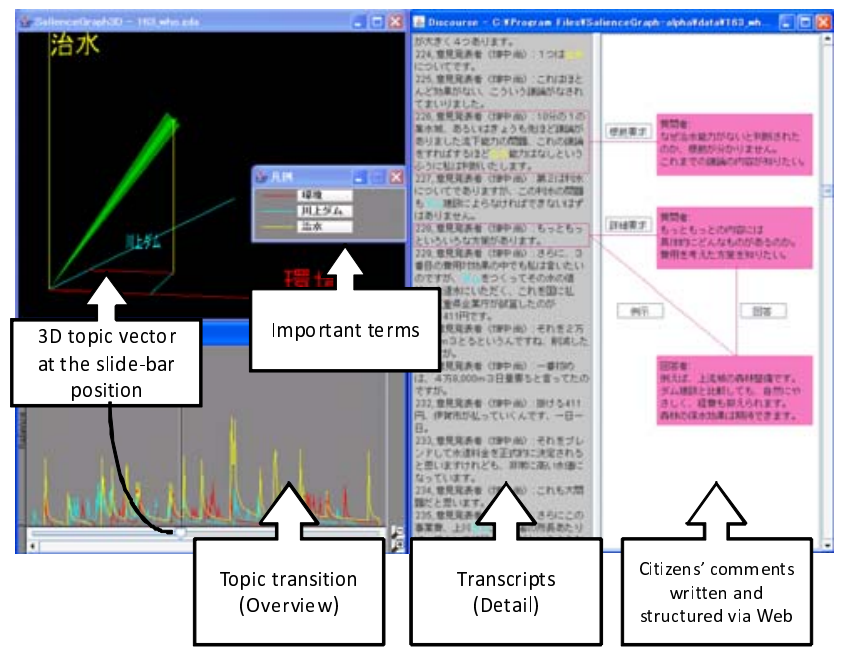

Fig. 2. SalienceGraph: Public debate browser for commenting via Web

and require-background. Since rhetorical structure indicates whether the answer matches the intention of the question, we presume that the novel relations are effective for facilitateing appropriate on-line debate. For example, when a negative conflict occurs in an on-line debate, we presume that users tend to provide an inappropriate answer, which does not satisfy the intention of the question. Hence, the design of the commenting interface assumes that users are motivated to specify a rhetorical relation tag to recieve their desired kind of answers.

\section{Salience-based Visualization of Topic Transition}

To reduce the time and effort needed to track and find arguments, we developed a visualizer for visualizing dynamic topic transition in a long meeting. Since the topic and joint attention dynamically change sentence by sentence, a metric for focus of attention on each term at each sentence needs to be designed (Figure 3). On the basis of this metric, a visualizer for topic transition in a meeting can be developed (Figure 4).

\subsection{Design of Salience Metric}

The horizontal axis of Figure 3 and the vertical axis of Figure 4 represent the salience of discourse entities [22]. We simply call it the "salience of terms". In our past research [2324], a metric for salience is designed as the reference probability under the assumption that a salient entity tends to be referred to in the subsequent utterance unit, and we use it here as well. Let $w$ be a word, $U_{i}$ be the current utterance unit, $U_{i+1}$ be the subsequent one, and $\operatorname{pre}\left(U_{i}\right)=$ $\left[U_{1}, \cdots, U_{i}\right]$ be the preceding discourse. Let $w^{\prime} \stackrel{\text { coref }}{\longrightarrow} w$ in $U_{i+1}$ denote that a word 
in $U_{i+1}$ has a coreference relation with $w$. The discourse salience of $w$ at $U_{i}$ is defined as the reference probability:

$$
\text { (Salience of } \begin{aligned}
\left.w \text { at } U_{i}\right) & =p\left(\exists w^{\prime} \stackrel{\text { coref }}{\longrightarrow} w \text { in } U_{i+1} \mid \operatorname{pre}\left(U_{i}\right)\right) \\
& =\operatorname{Pr}\left(w \mid \operatorname{pre}\left(U_{i}\right)\right)
\end{aligned}
$$

where $\operatorname{Pr}\left(w \mid \operatorname{pre}\left(U_{i}\right)\right)$ is a simplified notation for the reference probability. This probabilistic definition quantifies salience.

The consistency of the definition of salience with the centering theory, which is a theory of the continuity of the attentional state and pronominalization 22], is empirically verified using large English and Japanese corpora [23]. The reference probability $\operatorname{Pr}\left(w \mid \operatorname{pre}\left(U_{i}\right)\right)$ is statistically calculated from a corpus by logistic regression:

$$
\operatorname{Pr}\left(w \mid \operatorname{pre}\left(U_{i}\right)\right)=\frac{1}{1+\exp \left(-\left(\beta_{0}+\sum_{k} \beta_{k} \cdot \text { feat }_{k}\left(\left\langle w, U_{i}\right\rangle\right)\right)\right)},
$$

where feat $_{k}\left(\left\langle w, U_{i}\right\rangle\right)$ denotes feature values for the calculation, which is defined by using grammatical functions, part-of-speech, frequency, and recency [25]. The feature values are weighted by the recency effect [26] because the recent appearance of a target term should be emphasized over the old one 25]. The regression

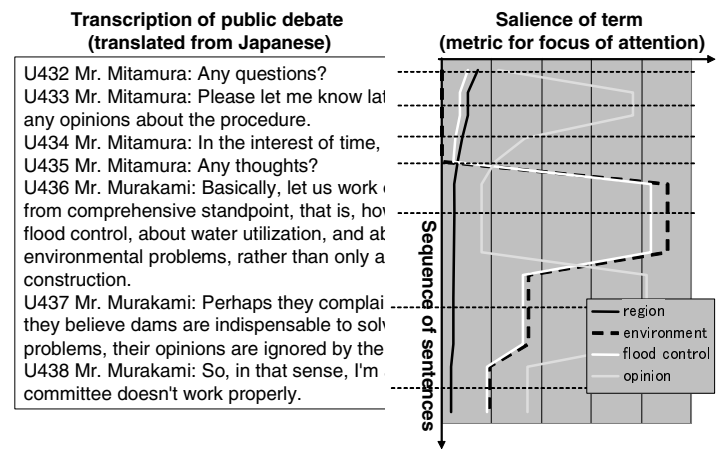

Fig. 3. Salience of term changes sentence by sentence

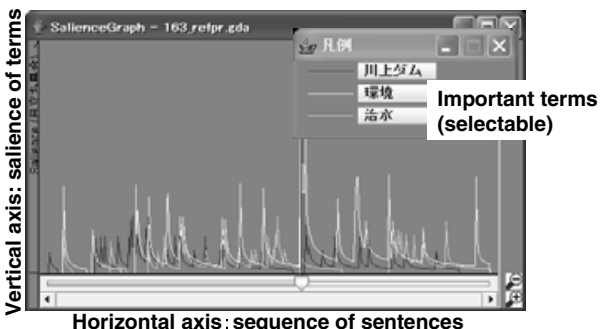

Fig. 4. Visualizing topic transition in long meeting to support overviewing, tracking, and finding arguments 
weight for each feat f $_{k}$ is denoted by $\beta_{k}$ in Equation (2). It is obtained from a corpus with the maximum-likelihood method.

Using this definition of salience enables us to deal with the transition on a sentence-by-sentence basis and to visualize the transition of topics (Figure 4). As shown in Figure 2, we developed a prototype of a debate browser, SalienceGraph, based on visualization scheme for topic transition, which is implemented using Java. It requires preprocessing for the transcription of a public debate as follows:

1. Analyze dependency structure using CaboCha, a Japanese dependency parser [27].

2. Annotate the result using CaboCha with GDA.

3. Calculate salience value $\operatorname{Pr}\left(w \mid \operatorname{pre}\left(U_{i}\right)\right)$ for each term $w$ and sentence $U_{i}$. The calculation target $\left\langle w, U_{i}\right\rangle$ is only such that $w$ appears in $\operatorname{pre}\left(U_{i}\right)$, the preceding context of $U_{i}$.

4. Annotate salience value $\operatorname{Pr}\left(w \mid \operatorname{pre}\left(U_{i}\right)\right)$ with GDA for each term $w$ and each sentence $U_{i}$.

\subsection{Design of Graphical User Interface (GUI)}

The GUI of SalienceGraph is designed based on Shneiderman's Visual Information-Seeking Mantra [28]. It supports an overview of transition of topics and finding an intended argument.

- Overview first: The user recieves an overview of transition in a long meeting using the topic transition window. The important terms can be chosen by using menus in the terms box. The menus have fifteen candidates of important terms with a high product of the total reference probability and inverse document frequency (IDF). The user can input other terms than the candidate ones into the text fields in the menu.

- Zoom and filter: When the user drags the slide bar under the topic transition window, the transcription window scrolls by being interlocked with the slide bar position. To read arguments about specific topics, e.g., "flood control" and "environment", the user chooses the terms he/she wishes to find from the menus and drag the slide bar to the position in which the terms have high salience values. The transcription window then scrolls to the argument about "flood control" and "environment", and the user can read her/his intended argument. Moreover, the transition window can be zoomed onto the intended argument.

- Details on demand: Thus, the user can find the detail of her/his intended argument. The movement of the $3 \mathrm{D}$ topic vector around the slide bar position also helps the user grasp the local transition of topics.

Furthermore, to help the user understand the background of the argument, information related to the topics needs to be provided at the slide bar position in the "Details on demand" phase. The next section describes a method for retrieving related information based on the salience of terms. 


\subsection{Retrieval of Topic-Related Information}

We developed a method for retrieving information related to the transient topics at the slide bar position in SalienceGraph [24]. Finer-grain information related to the transient topics can be retrieved based on the salience metric. A salience vector $\left[p\left(w_{1} \mid \operatorname{pre}\left(U_{i}\right)\right), \cdots, p\left(w_{n} \mid \operatorname{pre}\left(U_{i}\right)\right)\right]$, i.e., a vector comprising the salience of terms, represents topics at the moment of the sentence $U_{i}$. Because pre $\left(U_{i}\right)$ is calculated using the preceding context pre $\left(U_{i}\right)$, the vector can be used as a query vector representing the transient topic affected by the preceding context Since the salience vectors are calculated for each sentence, the nearest neighbor search based on salience vector enables finer-grain information retrieval.

Note that large calculation is necessary for the nearest neighbor search because the vocabulary size $n$ is in the tens of thousands. Therefore, we use a probabilistic Latent Semantic Analysis (pLSA) 29] to compress the dimensions of a salience vector and approximate nearest neighbor (ANN) [30] to search the nearest neighbors. The procedure is as follows.

1. For each meeting transcript and retrieval-targeted document, e.g., other meeting transcripts and newspaper articles, annotate dependency structure and salience values with GDA.

2. Extract $n$ terms whose product of the total reference probability and IDF are higher ranked from the records of meeting.

3. Compress the dimensions of $n$-dimensional salience vectors for each sentence in meetings and targeted documents by pLSA.

4. Retrieve candidate sentences with nearest neighbor vectors to provide topicrelated information by ANN on the basis of the compressed salience vectors.

5. Show the 5 best candidates using a tab window chosen by the user. In a tab, a candidate sentence and several preceding sentences are shown. The user can use them to choose the information they need from the candidates.

\section{$5 \quad$ Experimental Results}

\subsection{Evaluation of Salience Metric}

To evaluate our salience metric, the reference probability, we prepared two corpora in Japanese, which are annotated with GDA tags.

CSJ: Four interview dialogues from the Corpus of Spontaneous Japanese (CSJ) 31, which contain

- 1,780 utterance units (IPUs; inter-pause units),

- 6.92 morphemes per utterance unit, and

- 1,180 anaphora relations annotated manually.

Mainichi: 3,000 newspaper articles in Japanese from the Mainichi Shinbun for 1994 (GSK2004-A 32]), which contain

- 63,221 utterance units (predicate clauses), 37,340 sentences,

- 10.79 morphemes per utterance unit, and

- 86,541 anaphora relations annotated manually. 
We designed a metric evalSal $(m)$ to evaluate a target method $m$ for calculating salience.

$$
\operatorname{evalSal}(m)=\operatorname{cor}\left(\left[\operatorname{sal}_{m}\left(w \mid \operatorname{pre}\left(U_{i}\right)\right)\right]_{\left\langle w, U_{i}\right\rangle},\left[\operatorname{isRef}\left(w, U_{i+1}\right)\right]_{\left\langle w, U_{i}\right\rangle}\right),
$$

where isRef $\left(w, U_{i+1}\right)$ is the dummy variable defined above (i.e., 1 if $\exists w \stackrel{\text { ref }}{\longrightarrow}$ $e$ in $U_{i+1}$, otherwise 0$)$, and $\operatorname{cor}(x, y)$ denotes Pearson's correlation coefficient between $x$ and $y$.

We compared the evaluation scale, evalSal $(m)$, of our method for calculating the reference probability to that of the baseline method for the term frequency (TF) measured in an optimal rectangular window. The evaluation measures with the naive TF were 0.105 for $C S J$ and 0.301 for Mainichi. The evaluation measures with our proposed method ( 0.365 for $C S J$ and 0.368 for Mainichi) were greater. This means that our method can be used to more effectively predict whether the target entity is referred to in the subsequent $U_{i+1}$ than with the naive TF. The increase in effectiveness with our method was more significant for $C S J$ than for Mainichi. This indicates that handling spoken language needs more integration of the features than handling written language.

\section{Concluding Remarks}

We proposed SalienceGraph, a prototype system for supporting public debate with iterative alternation of face-to-face meetings and Web-based debates towards facilitating public involvement. To complement insufficiency of face-to-face debate, SalienceGraph includes a Web-based interface for commenting about meeting transcripts based on rhetorical structure theory. To support grasping of an overview of whole transition to track and to find intended arguments, we designed a salience metric and developed a novel method for visualizing topic transition. Our prototype system, SalienceGraph, was designed based on Visual Information-Seeking Mantra. The design visually helps the users to grasp an overview of topic transition and to find arguments from a lengthy debate.

Moreover, to support understanding of background of the arguments, we developed a method for salience-based retrieval of information related to transient topic specified by a user. The method for finer-grain retrieval does not need text segmentation because the salience vectors are annotated for each sentence. It is a natural expansion of the method for visualizing topic transition, which deals with topic transition and effects from the preceding context. Furthermore, we conducted experiments for retrieving topic-related information and instantiated the relevant information provided with our method. Our proposed framework reduces the amount of time and effort needed to aggregate and share arguments for public involvement.

As future work, we are planning to evaluate the efficiency of the Web-based commenting interface for sharing public concerns among citizens and stakeholders. 


\section{Acknowledgment}

The authors are grateful to Kiyoshi Kobayashi and Hayeong Jeong, who told us about the meeting records of the Yodo River Basin Committee, and Kôiti Hasida, who designed GDA tag set. This work was supported by Grant-in-Aid for Scientific Research (No. 19100003, No. 09152946).

\section{References}

1. Rowe, G., Frewer, L.: Public participation methods: A framework for evaluation. Science Technology Human Values 25(1), 3-29 (2000)

2. Renn, O., Webler, T., Rakel, H., Dienel, P., Johnson, B.: Public participation in decision making: A three-step procedure. Policy Sciences 26(3), 189-214 (1993)

3. Rowe, G., Frewer, L.: A typology of public engagement mechanisms. Science Technology Human Values 30(2), 251-290 (2005)

4. Nonaka, I., Toyama, R., Konno, N.: SECI, Ba and Leadership: a Unified Model of Dynamic Knowledge Creation. Long Range Planning 33(1), 5-34 (2000)

5. Yodo River Basin Committee: List of meeting minutes (2007) (in Japanese), http://www.yodoriver.org/doc_list/gijiroku.html

6. Malone, T., Laubacher, R., Introne, J., Klein, M., Abelson, H., Sterman, J., Olson, G.: The climate collaboratorium: Project overview. CCI Working Paper No. 2009003 (2009), http://cci.mit.edu/publications/CCIwp2009-03.pdf

7. Kirschner, P., Shum, S., Carr, C.: Visualizing Argumentation: Software Tools for Collaborative and Educational Sense-Making. Springer, Heidelberg (2003)

8. Verheij, B.: Artificial argument assistants for defeasible argumentation. Artificial Intelligence 150(1-2), 291-324 (2003)

9. Reed, C., Rowe, G.: Araucaria: Software for argument analysis, diagramming and representation. International Journal of AI Tools 13(4), 961-980 (2004)

10. Hasida, K.: Semantic Authoring and Semantic Computing. In: Sakurai, A., Hasida, K., Nitta, K. (eds.) JSAI 2003. LNCS (LNAI), vol. 3609, pp. 137-149. Springer, Heidelberg (2007)

11. van den Braak, S.W., van Oostendorp, H., Prakken, H., Vreeswijk, G.A.W.: A critical review of argument visualization tools: Do users become better reasoners? In: Workshop Notes of the ECAI 2006 Workshop on CMNA, pp. 67-75 (2006)

12. Bouamrane, M.M., Luz, S.: Navigating multimodal meeting recordings with the meeting miner. In: Larsen, H.L., Pasi, G., Ortiz-Arroyo, D., Andreasen, T., Christiansen, H. (eds.) FQAS 2006. LNCS (LNAI), vol. 4027, pp. 356-367. Springer, Heidelberg (2006)

13. Bouamrane, M.M., Luz, S.: Meeting browsing: State-of-the-art review. Multimedia Systems 12(4-5), 439-457 (2007)

14. Oinas-Kukkonen, H.: Debate browser - an argumentation tool for metaedit+ environment. In: Proceedings of the Seventh European Workshop on Next Generation of CASE Tools (NGCT '96), pp. 77-86 (1996)

15. Kamimaeda, N., Izumi, N., Hasida, K.: Evaluation of Participants' Contributions in Knowledge Creation Based on Semantic Authoring. The Learning Organization 14(3), 263-280 (1999)

16. Blei, D.M., Moreno, P.J.: Topic segmentation with an aspect hidden Markov model. In: Proceedings of SIGIR, pp. 343-348 (2001) 
17. Canny, J.F., Rattenbury, T.L.: A dynamic topic model for document segmentation. Technical report, EECS Department, University of California, Berkeley (2006)

18. Caracciolo, C., Hage, W.V., Rijke, M.D.: Towards topic driven access to full text documents. In: Proceedings of European Digital Library Conferences, pp. 495-500 (2004)

19. Jeong, H., Shiramatsu, S., Kobayashi, K., Hatori, T.: Discourse analysis of public debates using corpus linguistic methodologies. Journal of Computers 3(8), 58-68 (2008)

20. Hasida, K.: Global Document Annotation (GDA) (2004), http://i-content.org/GDA/

21. Mann, W., Thompson, S.: Rhetorical Structure Theory: Towards A Functional Theory of Text Organisation. Text 8(3), 243-281 (1988)

22. Grosz, B., Joshi, A., Weinstein, S.: Centering: A Framework for Modeling the Local Coherence of Discourse. Computational Linguistics 21(2), 203-226 (1995)

23. Shiramatsu, S., Komatani, K., Hasida, K., Ogata, T., Okuno, H.G.: A GameTheoretic Model of Referential Coherence and Its Empirical Verification Using Large Japanese and English Corpora. ACM Transactions on Speech and Language Processing (ACM-TSLP), Article 6, 5(3) (2008)

24. Shiramatsu, S., Kubota, Y., Komatani, K., Ogata, T., Takahashi, T., Okuno, H.: Visualization-based Approaches to Support Context Sharing towards Public Involment Support System. In: Opportunities and Challenges for Next-Generation Applied Intelligence. Studies in Computational Intelligence, vol. 214, pp. 111-117. Springer, Heidelberg (2009)

25. Shiramatsu, S., Komatani, K., Ogata, T., Okuno, H.G.: SalienceGraph: Visualizing Salience Dynamics of Written Discourse by Using Reference Probability and PLSA. In: Ho, T.-B., Zhou, Z.-H. (eds.) PRICAI 2008. LNCS (LNAI), vol. 5351, pp. 890902. Springer, Heidelberg (2008)

26. Murdock, B.B.: The Serial Position Effect in Free Recall. Journal of Experimental Psychology 64, 482-488 (1962)

27. Kudo, T., Matsumoto, Y.: Japanese dependency analysis using cascaded chunking. In: Proc. of CoNLL 2002, COLING 2002 Post-Conference Workshops, pp. 1-7 (2002)

28. Shneiderman, B.: Designing the User Interface: Strategies for Effective HumanComputer Interaction. Pearson Addison Wesley, London (1998)

29. Hofmann, T.: Probabilistic latent semantic analysis. In: Proc. of UAI 1999, pp. 289-296 (1999)

30. Arya, S., Mount, D.M.: Approximate Nearest Neighbor Queries in Fixed Dimensions. In: Proceedings of the Fourth Annual ACM/SIGACT-SIAM Symposium on Discrete Algorithms, pp. 271-280 (1993)

31. Maekawa, K.: Corpus of Spontaneous Japanese: Its Design and Evaluation. In: Proceedings of the ISCA \& SSPR 2003, pp. 7-12 (2003)

32. GSK (Gengo Shigen Kyokai): Linguistic resourse catalogue (in Japanese), http://www.gsk.or.jp/catalog.html 